\title{
Ulam Problem for the Cosine Addition Formula in Sato Hyperfunctions
}

\author{
Jaeyoung Chung' and Soon-Yeong Chung ${ }^{2}$ \\ ${ }^{1}$ Department of Mathematics, Kunsan National University, Kunsan 573-701, Republic of Korea \\ ${ }^{2}$ Department of Mathematics, Sogang University, Seoul 121-742, Republic of Korea
}

Correspondence should be addressed to Soon-Yeong Chung; sychung@sogang.ac.kr

Received 29 October 2014; Revised 21 December 2014; Accepted 8 January 2015

Academic Editor: Yuri Latushkin

Copyright (c) 2015 J. Chung and S.-Y. Chung. This is an open access article distributed under the Creative Commons Attribution License, which permits unrestricted use, distribution, and reproduction in any medium, provided the original work is properly cited.

We prove the Ulam problem for the cosine addition formula in the spaces of Schwartz distributions and Sato hyperfunctions with respect to bounded distributions and bounded hyperfunctions.

\section{Introduction}

The Ulam problem for functional equations goes back to 1940 when Ulam proposed the following [1].

Let $f$ be a mapping from a group $G_{1}$ to a metric group $G_{2}$ with metric $d(\cdot, \cdot)$ such that

$$
d(f(x y), f(x) f(y)) \leq \varepsilon .
$$

Then does there exist a group homomorphism $h$ and $\delta_{\epsilon}>0$ such that

$$
d(f(x), h(x)) \leq \delta_{\epsilon}
$$

for all $x \in G_{1}$ ?

This problem was solved affirmatively by Hyers under the assumption that $G_{2}$ is a Banach space (see Hyers [2], Hyers et al. [3]). In 1949-1951, this result was generalized by Aoki [4] and Bourgin [5, 6]. Since then Ulam problems of many other functional equations have been investigated [716]. Among the results, Székelyhidi has developed his idea of using invariant subspaces of functions defined on a group or semigroup in connection with Ulam problem for cosine functional equations $[17,18]$. As a direct consequence of the elegant results of Székelyhidi, it was obtained that if $f, g$ : $\mathbb{R}^{n} \rightarrow \mathbb{C}$ satisfy

$$
|g(x+y)-g(x) g(y)+f(x) f(y)| \leq M, \quad x, y \in \mathbb{R}^{n}
$$

for some $M>0$, then either there exist $\lambda_{1}, \lambda_{2} \in \mathbb{C}$ not both zero (either $\lambda_{1}$ or $\lambda_{2}$ is not zero), and $N>0$ such that

$$
\left|\lambda_{1} f(x)-\lambda_{2} g(x)\right| \leq N
$$

for all $x \in \mathbb{R}^{n}$, or

$$
g(x+y)-g(x) g(y)+f(x) f(y)=0
$$

for all $x, y \in \mathbb{R}^{n}$. Furthermore, the functions $f$ and $g$ satisfying both (3) and (4) are investigated.

In 1950, Laurent Schwartz introduced the theory of distributions in his monograph Théorie des distributions [19]. In this book Schwartz systematizes the theory of generalized functions, basing it on the theory of linear topological spaces, relates all the earlier approaches, and obtains many important results. After his elegant theory appeared, many important concepts and results on the classical spaces of functions have been generalized to the space of distributions. For example, positive functions and positive-definite functions have been generalized to positive distributions and positivedefinite distributions, respectively, and it is shown that every positive distribution is a positive measure [20, page 38] and every positive-definite distribution is the Fourier transform of positive measure $\mu$ such that $\int(1+|x|)^{-p} d \mu<\infty$ for some $p \geq 0$ [21, page 157], which is called Bochner-Schwartz theorem and is a natural generalization of the famous Bochner theorem stating that every positive-definite function is 
the Fourier transform of a positive finite measure. For other examples, the Paley-Wiener theorem has been generalized to the Paley-Wiener-Schwartz theorem which characterizes the distributions with bounded supports [20, page 181]. The main purpose of this paper is to prove a Hyers-Ulam type stability problem for the cosine functional equation in Schwartz distributions and Gelfand hyperfunctions. As a generalization of inequality (3), it is very natural to consider the inequality

$$
g(x+y)-g(x) g(y)+f(x) f(y) \in L^{\infty}\left(\mathbb{R}^{2 n}\right),
$$

where $f$ and $g$ are Lebesgue measurable functions and $L^{\infty}\left(\mathbb{R}^{2 n}\right)$ is the space of all bounded measurable functions defined in $\mathbb{R}^{2 n}$. Note that inequality (6) means that inequality (3) holds in almost everywhere sense. In [22-26], some stability problems of several functional equations including inequality (6) were considered in various spaces of generalized functions including Schwartz distributions. In [24-26], for example, replacing $f$ and $g$ by distributions $u$ and $v$ in inequality (6) we have considered

$$
v \circ S-v \otimes v+u \otimes u \in L^{\infty}\left(\mathbb{R}^{2 n}\right),
$$

where $S(x, y)=x+y, x, y \in \mathbb{R}^{n}$, 。 and $\otimes$ denote the pullback and tensor product of generalized functions. Inequality (7) cannot be considered as a complete formulation in the sense of generalized functions because the differences are assumed to be classical bounded measurable functions and all the previous results in [24-26] have the same formulations as in (7).

Due to Schwartz [19] the space $L^{\infty}\left(\mathbb{R}^{n}\right)$ of bounded measurable functions was generalized to the space $\mathscr{D}_{L^{\infty}}^{\prime}\left(\mathbb{R}^{n}\right)$ of bounded distributions. Taking the above generalizations into account, it is very natural and is a complete generalization to consider the stability problem for cosine functional equation in distributions and hyperfunctions $u, v$ with respect to bounded distributions and bounded hyperfunctions, respectively:

$$
\begin{aligned}
& v \circ S-v \otimes v+u \otimes u \in \mathscr{D}_{L^{\infty}}^{\prime}\left(\mathbb{R}^{2 n}\right), \\
& v \circ S-v \otimes v+u \otimes u \in \mathscr{A}_{L^{\infty}}^{\prime}\left(\mathbb{R}^{2 n}\right),
\end{aligned}
$$

where $\mathscr{D}_{L^{\infty}}^{\prime}\left(\mathbb{R}^{2 n}\right)$ and $\mathscr{A}_{L^{\infty}}^{\prime}\left(\mathbb{R}^{2 n}\right)$ are the spaces of bounded distributions and bounded hyperfunctions, respectively, and $S$, ॰ and $\otimes$ are the same as in (7). For some related results in Schwartz distributions, we refer the reader to $[19,20,22,23$, 27-29].

The main tool of solving (8) and (9) is the heat kernel method initiated by Matsuzawa which represents the generalized functions as the initial values of solutions of the heat equation with some growth conditions [30-33].

\section{Spaces of Distributions and Hyperfunctions and Some Preliminary Results}

We first introduce the spaces $\mathcal{S}^{\prime}$ of Schwartz tempered distributions and the space $\mathscr{G}^{\prime}$ of Gelfand hyperfunctions (see $[19-21,33,34]$ for more details of these spaces). We use the notations $|\alpha|=\alpha_{1}+\cdots+\alpha_{n}, \alpha !=\alpha_{1} ! \cdots \alpha_{n} !,|x|=$ $\sqrt{x_{1}^{2}+\cdots x_{n}^{2}}, x^{\alpha}=x_{1}^{\alpha_{1}} \cdots x_{n}^{\alpha_{n}}$, and $\partial^{\alpha}=\partial_{1}^{\alpha_{1}} \cdots \partial_{n}^{\alpha_{n}}$, for $x=$ $\left(x_{1}, \ldots, x_{n}\right) \in \mathbb{R}^{n}, \alpha=\left(\alpha_{1}, \ldots, \alpha_{n}\right) \in \mathbb{N}_{0}^{n}$, where $\mathbb{N}_{0}$ is the set of nonnegative integers and $\partial_{j}=\partial / \partial x_{j}$.

Definition 1 (see [19]). One denotes by $\mathcal{S}$ or $\mathcal{S}\left(\mathbb{R}^{n}\right)$ the Schwartz space of all infinitely differentiable functions $\varphi$ in $\mathbb{R}^{n}$ such that

$$
\|\varphi\|_{\alpha, \beta}=\sup _{x}\left|x^{\alpha} \partial^{\beta} \varphi(x)\right|<\infty
$$

for all $\alpha, \beta \in \mathbb{N}_{0}^{n}$, equipped with the topology defined by the seminorms $\|\cdot\|_{\alpha, \beta}$. The elements of $\mathcal{S}$ are called rapidly decreasing functions and the elements of the dual space $\mathcal{S}^{\prime}$ are called tempered distributions.

Definition 2 (see $[21,34]$ ). One denotes by $\mathscr{G}$ or $\mathscr{G}\left(\mathbb{R}^{n}\right)$ the Gelfand space of all infinitely differentiable functions $\varphi$ in $\mathbb{R}^{n}$ such that

$$
\|\varphi\|_{h, k}=\sup _{x \in \mathbb{R}^{n}, \alpha, \beta \in \mathbb{N}_{0}^{n}} \frac{\left|x^{\alpha} \partial^{\beta} \varphi(x)\right|}{h^{|\alpha|} k^{|\beta|} \alpha !^{1 / 2} \beta !^{1 / 2}}<\infty
$$

for some $h, k>0$. One says that $\varphi_{j} \rightarrow 0$ as $j \rightarrow \infty$ if $\left\|\varphi_{j}\right\|_{h, k} \rightarrow 0$ as $j \rightarrow \infty$ for some $h, k$ and denotes by $\mathscr{G}^{\prime}$ the strong dual space of $\mathscr{G}$ and calls its elements Gelfand hyperfunctions.

As a generalization of the space $L^{\infty}$ of bounded measurable functions, Schwartz introduced the space $\mathscr{D}_{L^{\infty}}^{\prime}$ of bounded distributions as a subspace of tempered distributions.

Definition 3 (see [19]). One denotes by $\mathscr{D}_{L^{1}}\left(\mathbb{R}^{n}\right)$ the space of smooth functions on $\mathbb{R}^{n}$ such that $\partial^{\alpha} \varphi \in L^{1}\left(\mathbb{R}^{n}\right)$ for all $\alpha \in \mathbb{N}_{0}^{n}$ equipped with the topology defined by the countable family of seminorms

$$
\|\varphi\|_{m}=\sum_{|\alpha| \leq m}\left\|\partial^{\alpha} \varphi\right\|_{L^{1}}, \quad m \in \mathbb{N}_{0} .
$$

One denotes by $\mathscr{D}_{L^{\infty}}^{\prime}$ the strong dual space of $\mathscr{D}_{L^{1}}$ and calls its elements bounded distributions.

Generalizing bounded distributions the space $\mathscr{A}_{L^{\infty}}^{\prime}$ of bounded hyperfunctions has been introduced as a subspace of $\mathscr{G}^{\prime}$.

Definition 4 (see [32]). One denotes by $\mathscr{A}_{L^{1}}$ the space of smooth functions on $\mathbb{R}^{n}$ satisfying

$$
\|\varphi\|_{h}=\sup _{\alpha} \frac{\left\|\partial^{\alpha} \varphi\right\|_{L^{1}}}{h^{|\alpha|} \alpha !}<\infty
$$

for some constant $h>0$. One says that $\varphi_{j} \rightarrow 0$ in $\mathscr{A}_{L^{1}}$ as $j \rightarrow \infty$ if there is a positive constant $h$ such that

$$
\sup _{\alpha} \frac{\left\|\partial^{\alpha} \varphi_{j}\right\|_{L^{1}}}{h^{|\alpha|} \alpha !} \longrightarrow 0 \text { as } j \longrightarrow \infty \text {. }
$$

One denotes by $\mathscr{A}_{L^{\infty}}^{\prime}$ the strong dual space of $\mathscr{A}_{L^{1}}$. 

hold:

It is well known that the following topological inclusions

$$
\begin{array}{cl}
\mathscr{G} \hookrightarrow \mathcal{S} \hookrightarrow \mathscr{D}_{L^{1}}, & \mathscr{D}_{L^{\infty}}^{\prime} \hookrightarrow \mathcal{S}^{\prime} \hookrightarrow \mathscr{G}^{\prime}, \\
\mathscr{G} \hookrightarrow \mathscr{A}_{L^{1}} \hookrightarrow \mathscr{D}_{L^{1}}, & \mathscr{D}_{L^{\infty}}^{\prime} \hookrightarrow \mathscr{A}_{L^{\infty}}^{\prime} \hookrightarrow \mathscr{G}^{\prime} .
\end{array}
$$

It is known that the space $\mathscr{G}\left(\mathbb{R}^{n}\right)$ consists of all infinitely differentiable functions $\varphi(x)$ on $\mathbb{R}^{n}$ which can be extended to an entire function on $\mathbb{C}^{n}$ satisfying

$$
|\varphi(x+i y)| \leq C \exp \left(-a|x|^{2}+b|y|^{2}\right), \quad x, y \in \mathbb{R}^{n}
$$

for some $a, b, C>0$ (see [34]).

Definition 5. Let $u_{j} \in \mathscr{G}^{\prime}\left(\mathbb{R}^{n_{j}}\right)$ for $j=1,2$. Then the tensor product $u_{1} \otimes u_{2}$ of $u_{1}$ and $u_{2}$, defined by

$$
\left\langle u_{1} \otimes u_{2}, \varphi\left(x_{1}, x_{2}\right)\right\rangle=\left\langle u_{1},\left\langle u_{2}, \varphi\left(x_{1}, x_{2}\right)\right\rangle\right\rangle
$$

for $\varphi\left(x_{1}, x_{2}\right) \in \mathscr{G}\left(\mathbb{R}^{n_{1}} \times \mathbb{R}^{n_{2}}\right)$, belongs to $\mathscr{G}^{\prime}\left(\mathbb{R}^{n_{1}} \times \mathbb{R}^{n_{2}}\right)$.

For the proof of our theorems we employ the $n$ dimensional heat kernel $E_{t}(x)$ given by

$$
E_{t}(x)=(4 \pi t)^{-n / 2} \exp \left(-\frac{|x|^{2}}{4 t}\right), \quad t>0 .
$$

In view of (16), we can see that the heat kernel $E_{t}$ belongs to the Gelfand space $\mathscr{G}\left(\mathbb{R}^{n}\right)$ for each $t>0$. Thus, for each $u \in \mathscr{G}^{\prime}\left(\mathbb{R}^{n}\right)$, the convolution $\left(u * E_{t}\right)(x):=\left\langle u_{y}, E_{t}(x-y)\right\rangle$ is well defined. We call $\left(u * E_{t}\right)(x)$ the Gauss transform of $u$. From now on we denote by $\widetilde{u}(x, t)$ the Gauss transform of $u$. It is well known that the Gauss transform $\widetilde{u}(x, t)$ is a smooth solution of the heat equation such that $\widetilde{u}(x, t) \rightarrow u$ in weak star topology as $t \rightarrow 0^{+}$; that is,

$$
\langle u, \varphi\rangle=\lim _{t \rightarrow 0^{+}} \int \widetilde{u}(x, t) \varphi(x) d x
$$

for all $\varphi \in \mathscr{G}$.

For the proof of our main result the following results are very useful. The proof of Theorem 2.3 of [35] works even when $p=\infty$; that is, we obtain the following.

Lemma 6 (see [35]). The Gauss transform $\widetilde{u}(x, t):=(u *$ $E)(x, t)$ of $u \in \mathscr{D}_{L^{\infty}}^{\prime}\left(\mathbb{R}^{n}\right)$ is a smooth solution of the heat equation $\left(\Delta-\partial / \partial_{t}\right) \tilde{u}=0$ satisfying the following.

(i) There exist constants $C>0, N \geq 0$ such that

$$
|\widetilde{u}(x, t)| \leq C t^{-N} \quad \forall x \in \mathbb{R}^{n}, t>0 .
$$

(ii) $\tilde{u}(x, t) \rightarrow u$ as $t \rightarrow 0^{+}$in the sense that, for every $\varphi \in \mathscr{D}_{L^{1}}$

$$
\langle u, \varphi\rangle=\lim _{t \rightarrow 0^{+}} \int \tilde{u}(x, t) \varphi(x) d x .
$$

Conversely, every smooth solution $\widetilde{u}(x, t)$ of the heat equation satisfying the estimate (20) can be uniquely expressed as $\widetilde{u}(x, t)=(u * E)(x, t)$ for some $u \in \mathscr{D}_{L^{\infty}}^{\prime}\left(\mathbb{R}^{n}\right)$.
The following lemma is a special case of Theorem 3.5 of [32] when $p=\infty$ where the space $\mathscr{A}_{L^{\infty}}^{\prime}\left(\mathbb{R}^{n}\right)$ is denoted by $\mathscr{B}_{L^{\infty}}\left(\mathbb{R}^{n}\right)$.

Lemma 7 (see [32]). The Gauss transform $\widetilde{u}(x, t):=(u *$ $E)(x, t)$ of $u \in \mathscr{A}_{L^{\infty}}^{\prime}\left(\mathbb{R}^{n}\right)$ is a smooth solution of the heat equation $\left(\Delta-\partial / \partial_{t}\right) \tilde{u}=0$ satisfying the following.

(i) For every $\epsilon>0$ there exists a constant $C_{\epsilon}>0$ such that

$$
|\widetilde{u}(x, t)| \leq C_{\epsilon} e^{\epsilon / t} \quad \forall x \in \mathbb{R}^{n}, t>0 .
$$

(ii) $\widetilde{u}(x, t) \rightarrow u$ as $t \rightarrow 0^{+}$in the sense that, for every $\varphi \in \mathscr{A}_{L^{1}}$,

$$
\langle u, \varphi\rangle=\lim _{t \rightarrow 0^{+}} \int \widetilde{u}(x, t) \varphi(x) d x .
$$

Conversely, every smooth solution $\widetilde{u}(x, t)$ of the heat equation satisfying the estimate (22) can be uniquely expressed as $\widetilde{u}(x, t)=(u * E)(x, t)$ for some $u \in \mathscr{D}_{L^{\infty}}^{\prime}\left(\mathbb{R}^{n}\right)$.

The following structure theorem for bounded distributions is well known.

Lemma 8 (see [19]). Every $u \in \mathscr{D}_{L^{\infty}}^{\prime}\left(\mathbb{R}^{n}\right)$ can be expressed as

$$
u=\sum_{|\alpha| \leq p} \partial^{\alpha} f_{\alpha}
$$

for some $p \in \mathbb{N}_{0}$, where $f_{\alpha}$ are bounded measurable functions on $\mathbb{R}^{n}$. Equality (24) implies that

$$
\langle u, \varphi\rangle=\sum_{|\alpha| \leq p}(-1)^{|\alpha|} \int f_{\alpha}(x) \partial^{\alpha} \varphi(x) d x
$$

for all $\varphi \in \mathscr{D}_{L^{1}}$.

As a special case of Theorem 3.4 of [32] when $p=\infty$ where the space $\mathscr{A}_{L^{\infty}}^{\prime}\left(\mathbb{R}^{n}\right)$ is denoted by $\mathscr{B}_{L^{\infty}}\left(\mathbb{R}^{n}\right)$ we obtain the following.

Lemma 9 (see [32]). Every $u \in \mathscr{A}_{L^{\infty}}^{\prime}\left(\mathbb{R}^{n}\right)$ can be expressed by

$$
u=\left(\sum_{k=0}^{\infty} a_{k} \Delta^{k}\right) g+h
$$

where $\Delta$ denotes the Laplacian, $g, h$ are bounded continuous functions on $\mathbb{R}^{n}$, and $a_{k}, k=0,1,2, \ldots$, satisfy the following estimates; for every $L>0$ there exists $C>0$ such that

$$
\left|a_{k}\right| \leq \frac{C L^{k}}{k !^{2}}
$$

for all $k=0,1,2, \ldots$

The following properties of the heat kernel will be useful which can be found in [33]. 
Proposition 10 (see [33]). For each $t>0, E_{t}(\cdot)$ is an entire function and the following estimate holds; there exists $C>0$ such that

$$
\left|\partial_{x}^{\alpha} E_{t}(x)\right| \leq C^{|\alpha|} t^{-(n+|\alpha|) / 2} \alpha !^{1 / 2} \exp \left(-\frac{|x|^{2}}{8 t}\right) .
$$

Also for each $t, s>0$ one has

$$
\left(E_{t} * E_{s}\right)(x):=\int E_{t}(x-y) E_{s}(y) d y=E_{t+s}(x) .
$$

\section{Stability Problems with Time Variable}

The main tools of the proof of our main result are based on the heat kernel method initiated by Matsuzawa [33] (see Lemmas 6 and 7 in Section 2) and the structure theorems (see Lemmas 8 and 9). Making use of the heat kernel and the structure theorems we convert (8) and (9) to the following classical stability problems for the Gauss transforms $\widetilde{u}, \widetilde{v}$ : $\mathbb{R}^{n} \times(0, \infty) \rightarrow \mathbb{C}$ of $u, v$, respectively:

there exist $C>0, N>0, d>0$ such that

$$
\begin{aligned}
& |\widetilde{v}(x+y, t+s)-\widetilde{v}(x, t) \widetilde{v}(y, s)+\widetilde{u}(x, t) \tilde{u}(y, s)| \\
& \leq C\left(\frac{1}{t}+\frac{1}{s}\right)^{N}+d, \quad \forall x, y \in G, t, s>0 ;
\end{aligned}
$$

for every $\epsilon>0$, there exists $C_{\epsilon}>0$ such that

$$
\begin{aligned}
& |\widetilde{v}(x+y, t+s)-\widetilde{v}(x, t) \widetilde{v}(y, s)+\widetilde{u}(x, t) \widetilde{u}(y, s)| \\
& \quad \leq C_{\epsilon} e^{\epsilon(1 / t+1 / s)}, \quad \forall x, y \in G, t, s>0 .
\end{aligned}
$$

In this section we prove the stability problems of inequality (30) with more general settings: let $f, g: G \times(0, \infty) \rightarrow \mathbb{C}$ with $\langle G,+\rangle$ an Abelian group and let $N$ be a nonnegative real number. We consider the following stability problems, respectively:

there exist $C>0, d>0$ such that

$$
\begin{aligned}
& |g(x+y, t+s)-g(x, t) g(y, s)+f(x, t) f(y, s)| \\
& \quad \leq C\left(\frac{1}{t}+\frac{1}{s}\right)^{N}+d, \quad \forall x, y \in G, t, s>0
\end{aligned}
$$

for every $\epsilon>0$, there exists $C_{\epsilon}>0$ such that

$$
\begin{aligned}
& |g(x+y, t+s)-g(x, t) g(y, s)+f(x, t) f(y, s)| \\
& \leq C_{\epsilon} e^{\epsilon(1 / t+1 / s)}, \quad \forall x, y \in G, t, s>0 .
\end{aligned}
$$

From now on, a function $A$ from a semigroup $\langle S,+\rangle$ to the field $\mathbb{C}$ of complex numbers is said to be an additive function provided $A(x+y)=A(x)+A(y)$ for all $x, y \in S$ and $m: S \rightarrow \mathbb{C}$ is said to be an exponential function provided $m(x+y)=m(x) m(y)$ for all $x, y \in S$. We introduce the following conditions on $f: G \times(0, \infty) \rightarrow \mathbb{C}$ and $N \geq 0$ :

there exist $C>0, d>0$ such that

$|f(x, t)| \leq C t^{-N}+d, \quad \forall x \in G, t>0 ;$

for every $\epsilon>0$, there exists $C_{\epsilon}>0$

which depends on $\epsilon$ such that

$$
|f(x, t)| \leq C_{\epsilon} e^{\epsilon / t}, \quad \forall x \in G, t>0 .
$$

For the proof of the following lemma we refer to [36, Lemma 2.2].

Lemma 11. Let $f, g: G \times(0, \infty) \rightarrow \mathbb{C}$ satisfy the inequality; for each $y \in G$ and $s>0$ there exist positive constants $C=$ $C(y, s)$ and $d=d(y, s)$ (resp., for each $y \in G, s>0$, and $\epsilon>0$ there exists a positive constant $\left.C_{\epsilon}=C_{\epsilon}(y, s)\right)$ such that

$$
\begin{gathered}
|f(x+y, t+s)-f(x, t) g(y, s)| \\
\leq C t^{-N}+d \quad\left(\text { resp., } C_{\epsilon} e^{\epsilon / t}\right)
\end{gathered}
$$

for all $x \in G, t>0$. Then either $f$ satisfies (33) (resp., (34)) or $g$ is an exponential function.

For the proof of the following lemma we refer to [36, Lemma 2.3].

Lemma 12. Let $g: G \times(0, \infty) \rightarrow \mathbb{C}$ be a nonzero exponential function satisfying (33) (resp., (34)). Then g can be written in the form

$$
g(x, t)=m_{1}(x) m_{2}(t),
$$

where $m_{1}$ is an exponential function on $G$ satisfying $\left|m_{1}(x)\right|=$ 1 for all $x \in G$ and $m_{2}$ is an exponential function on $(0, \infty)$ satisfying $0<\left|m_{2}(t)\right| \leq 1$ for all $t>0$.

For the proof of the following lemma we refer to [36, Lemma 2.4].

Lemma 13. Let $m: G \times(0, \infty) \rightarrow \mathbb{C}$ be a nonzero exponential function satisfying (33) (resp., (34)). Suppose that $g: G \times$ $(0, \infty) \rightarrow \mathbb{C}$ satisfies the inequality; there exist $C>0$ and $d>0$ (resp., for every $\epsilon>0$ there exists $C_{\epsilon}>0$ ) such that

$$
\begin{aligned}
& |g(x+y, t+s)-g(x, t) m(y, s)-m(x, t) g(y, s)| \\
& \leq C\left(\frac{1}{t}+\frac{1}{s}\right)^{N}+d \quad\left(\text { resp., } C_{\epsilon} e^{\epsilon(1 / t+1 / s)}\right)
\end{aligned}
$$

for all $x, y \in G, t, s>0$. Then one has

$$
\begin{gathered}
m(x, t)=m_{1}(x) m_{2}(t) \\
g(x, t)=A(x) m_{1}(x) m_{2}(t)+2 g\left(0, \frac{t}{2}\right) m_{2}\left(\frac{t}{2}\right) m_{1}(x) \\
+R(x, t)
\end{gathered}
$$


where $m_{1}$ and $m_{2}$ are exponential functions on $G$ and $(0, \infty)$, respectively, such that $\left|m_{1}(x)\right|=1$ for all $x \in G$ and $0<$ $\left|m_{2}(t)\right| \leq 1$ for all $t>0$, and $A(x)$ is an additive function on $G$ and $R$ is a function satisfying (33) (resp., (34)).

Theorem 14. Let $g, f: G \times(0, \infty) \rightarrow \mathbb{C}$ satisfy (31) (resp., (32)). Then $(g, f)$ satisfies one of the following:

(i) both $g$ and $f$ satisfy (33) (resp., (34));

(ii) $g$ is an exponential function and $f$ satisfies (33) (resp., (34));

(iii) $g(x, t)=(m(x, t)+\lambda R(x, t)) /\left(1-\lambda^{2}\right), f(x, t)=$ $(\lambda m(x, t)+R(x, t)) /\left(1-\lambda^{2}\right)$, where $\lambda(\neq \pm 1) \in \mathbb{C}, m$ is a nonzero exponential function, and $R$ is a function satisfying (33) (resp., (34));

(iv) $g(x, t)=(A(x)+1) m_{1}(x) m_{2}(t)+2 g(0, t / 2) m_{2}(t /$ 2) $m_{1}(x)+R(x, t), \pm f(x, t)=A(x) m_{1}(x) m_{2}(t)+$ $2 g(0, t) m_{2}(t / 2) m_{1}(x)+R(x, t)$, where $A(x)$ is an additive function, $m_{1}$ and $m_{2}$ are exponential functions on $G$ and $(0, \infty)$, respectively, such that $\left|m_{1}(x)\right|=1$ for all $x \in G$ and $0<\left|m_{2}(t)\right| \leq 1$ for all $t>0$, and $R$ is a function satisfying (33) (resp., (34));

(v) $g(x+y, t+s)-g(x, t) g(y, s)+f(x, t) f(y, s)=0$ for all $x, y \in G, t, s>0$.

Proof. We first prove that either there exist $\lambda_{1}, \lambda_{2} \in \mathbb{C}$, not both zero, and $C>0, d>0$ (resp., for every $\epsilon>0$, there exists $C_{\epsilon}>0$ ) such that

$$
\left|\lambda_{1} f(x, t)-\lambda_{2} g(x, t)\right| \leq C t^{-N}+d \quad\left(\text { resp., } C_{\epsilon} e^{\epsilon / t}\right)
$$

or

$$
g(x+y, t+s)-g(x, t) g(y, s)+f(x, t) f(y, s)=0
$$

for all $x, y \in G, t, s>0$.

Suppose that inequality (39) holds only when $\lambda_{1}=\lambda_{2}=$ 0 . Then we can choose $y_{1} \in G$ and $s_{1}>0$ satisfying $f\left(y_{1}, s_{1}\right) \neq$ 0 . Let

$$
\begin{aligned}
F(x, y, t, s)= & g(x+y, t+s)-g(x, t) g(y, s) \\
& +f(x, t) f(y, s) .
\end{aligned}
$$

Then we can write

$$
\begin{aligned}
f(x, t)= & k_{1} g(x, t)+k_{2} g\left(x+y_{1}, t+s_{1}\right) \\
& -k_{2} F\left(x, y_{1}, t, s_{1}\right),
\end{aligned}
$$

where $k_{1}=g\left(y_{1}, s_{1}\right) / f\left(y_{1}, s_{1}\right)$ and $k_{2}=-1 / f\left(y_{1}, s_{1}\right)$. Using (41) and (42) we have

$$
\begin{aligned}
& g((x+y)+z,(t+s)+r) \\
& =g(x+y, t+s) g(z, r)-f(x+y, t+s) f(z, r) \\
& +F(x+y, z, t+s, r) \\
& =(g(x, t) g(y, s)-f(x, t) f(y, s) \\
& +F(x, y, t, s)) g(z, r) \\
& -\left(k_{1} g(x+y, t+s)\right. \\
& +k_{2} g\left(x+y+y_{1}, t+s+s_{1}\right) \\
& \left.-k_{2} F\left(x+y, y_{1}, t+s, s_{1}\right)\right) f(z, r) \\
& +F(x+y, z, t+s, r) \\
& =(g(x, t) g(y, s)-f(x, t) f(y, s) \\
& +F(x, y, t, s)) g(z, r) \\
& -k_{1}(g(x, t) g(y, s)-f(x, t) f(y, s) \\
& +F(x, y, t, s)) f(z, r) \\
& -k_{2}\left(g(x, t) g\left(y+y_{1}, s+s_{1}\right)\right. \\
& -f(x, t) f\left(y+y_{1}, s+s_{1}\right) \\
& \left.+F\left(x, y+y_{1}, t, s+s_{1}\right)\right) f(z, r) \\
& +k_{2} F\left(x+y, y_{1}, t+s, s_{1}\right) f(z, r) \\
& +F(x+y, z, t+s, r) .
\end{aligned}
$$

On the other hand, we can write

$$
\begin{aligned}
g(x+ & (y+z), t+(s+r)) \\
= & g(x, t) g(y+z, s+r)-f(x, t) f(y+z, s+r) \\
& +F(x, y+z, t, s+r) .
\end{aligned}
$$

By equating the right hand sides of (43) and (44) we have

$$
\begin{aligned}
& g(x, t)\left(g(y, s) g(z, r)-k_{1} g(y, s) f(z, r)\right. \\
&\left.-k_{2} g\left(y+y_{1}, s+s_{1}\right) f(z, r)-g(y+z, s+r)\right) \\
&+f(x, t)\left(-f(y, s) g(z, r)+k_{1} f(y, s) f(z, r)\right. \\
&+k_{2} f\left(y+y_{1}, s+s_{1}\right) f(z, r) \\
&\quad+f(y+z, s+r)) \\
&=-F(x, y, t, s) g(z, r)+k_{1} F(x, y, t, s) f(z, r) \\
&+k_{2} F\left(x, y+y_{1}, t, s+s_{1}\right) f(z, r) \\
&-k_{2} F\left(x+y, y_{1}, t+s, s_{1}\right) f(z, r) \\
&-F(x+y, z, t+s, r)+F(x, y+z, t, s+r) .
\end{aligned}
$$


If we fix $y, s, z, r$ in (45), the right hand side of (45) satisfies (33) (resp., (34)). Thus, our assumption implies

$$
\begin{aligned}
F(x, y+z, t, s+r)-F(x+y, z, t+s, r) \\
=F(x, y, t, s) g(z, r) \\
\quad+\left(-k_{1} F(x, y, t, s)-k_{2} F\left(x, y+y_{1}, t, s+s_{1}\right)\right. \\
\left.\quad+k_{2} F\left(x+y, y_{1}, t+s, s_{1}\right)\right) f(z, r) .
\end{aligned}
$$

Now if we fix $x, y, t, s$ in (46), the left hand side of (46) satisfies (33) (resp., (34)) as a function of $z$ and $r$. Thus our assumption implies $F \equiv 0$. Thus we have case (v).

From now on, we assume that inequality (39) holds: if $f$ satisfies (33) (resp., (34)), then cases (i) and (ii) follow immediately from Lemma 11. It is easy to see that if $f$ does not satisfy (33) (resp., (34)), then neither does $g$. For this case, inequality (39) implies $\lambda_{1} \lambda_{2} \neq 0$ and we can write

$$
f(x, t)=\lambda g(x, t)+R(x, t)
$$

for some $\lambda(\neq 0) \in \mathbb{C}$ and a function $R$ satisfying (33) (resp., (34)).

Putting (47) in (31) (resp., (32)) using the triangle inequality and fixing $y, s$ we have

$$
\begin{aligned}
& \left|g(x+y, t+s)-g(x, t)\left(\left(1-\lambda^{2}\right) g(y, s)-\lambda R(y, s)\right)\right| \\
& \quad \leq|R(x, t)[R(y, s)+\lambda g(y, s)]|+C\left(\frac{1}{t}+\frac{1}{s}\right)^{N}+d \\
& \quad \leq C^{\prime} t^{-N}+d^{\prime} \quad\left(\text { resp., } C_{\epsilon}^{\prime} e^{\epsilon / t}\right)
\end{aligned}
$$

for all $x \in G, t>0$ and for some positive constants $C^{\prime}$ and $d^{\prime}$ (resp., for every $\epsilon>0$ there exists a positive constant $C_{\epsilon}^{\prime}$ ). Using Lemma 11, we have

$$
\left(1-\lambda^{2}\right) g(y, s)-\lambda R(y, s)=m(y, s)
$$

for all $y \in G, s>0$, where $m$ is an exponential function on $G \times(0, \infty)$.

Now if $\lambda \neq \pm 1$, case (iii) follows. If $\lambda= \pm 1$, then we have

$$
\begin{gathered}
m(x, t)=\mp R(x, t), \\
\pm f(x, t)=g(x, t)-m(x, t)
\end{gathered}
$$

for all $x \in G, t>0$. Viewing (50) and using Lemma 12 we have

$$
m(x, t)=m_{1}(x) m_{2}(t),
$$

where $\left|m_{1}(x)\right|=1,0<\left|m_{2}(t)\right| \leq 1$ for all $x \in G, t>0$. Putting (51) in (31) (resp., (32)) and using the triangle inequality we have

$$
\begin{aligned}
& |g(x+y, t+s)-g(x, t) m(y, s)-g(y, s) m(x, t)| \\
& \quad \leq|m(x, t) m(y, s)|+C\left(\frac{1}{t}+\frac{1}{s}\right)^{N}+d \\
& \left.\quad \leq C\left(\frac{1}{t}+\frac{1}{s}\right)^{N}+d+1 \quad \text { (resp., } C_{\epsilon}^{\prime} e^{\epsilon(1 / t+1 / s)}\right)
\end{aligned}
$$

for all $x, y \in G, t, s>0$. By Lemma 13 we have

$$
\begin{aligned}
g(x, t)= & A(x) m_{1}(x) m_{2}(t) \\
& +2 g\left(0, \frac{t}{2}\right) m_{2}\left(\frac{t}{2}\right) m_{1}(x)+R_{1}(x, t),
\end{aligned}
$$

where $A(x)$ is an additive function and $R_{1}(x, t)$ is a function satisfying (33) (resp., (34)). Thus it follows from (51) and (54) that

$$
\begin{aligned}
\pm f(x, t)= & (A(x)-1) m_{1}(x) m_{2}(t) \\
& +2 g\left(0, \frac{t}{2}\right) m_{2}\left(\frac{t}{2}\right) m_{1}(x)+R_{1}(x, t)
\end{aligned}
$$

for all $x \in G, t>0$. Replacing $R_{1}-m$ by $R$ we obtain (iv). This completes the proof.

From Theorem 14 we have the following.

Corollary 15. Let $g, f: \mathbb{R}^{n} \times(0, \infty) \rightarrow \mathbb{C}$ be continuous functions satisfying (31) (resp., (32)). Then $(g, f)$ satisfies one of the following:

(i) both $g$ and $f$ satisfy (33) (resp., (34));

(ii) $g(x, t)=e^{c \cdot x+b t}$ and $f$ satisfies (33) (resp., (34)), where $c \in \mathbb{C}^{n}, b \in \mathbb{C}$

(iii) $g(x, t)=\left(e^{c \cdot x+b t}+\lambda R(x, t)\right) /\left(1-\lambda^{2}\right), f(x, t)=$ $\left(\lambda e^{c \cdot x+b t}+R(x, t)\right) /\left(1-\lambda^{2}\right)$, where $c \in \mathbb{C}^{n}, b, \lambda(\neq \pm 1) \epsilon$ $\mathbb{C}$, and $R$ is a function satisfying (33) (resp., (34));

(iv) $g(x, t)=(a \cdot x+1) e^{i c \cdot x+b t}+2 g(0, t / 2) e^{i c \cdot x+(1 / 2) b t}+$ $R(x, t), \pm f(x, t)=a \cdot x e^{i c \cdot x+b t}+2 g(0, t / 2) e^{i c \cdot x+(1 / 2) b t}+$ $R(x, t)$, where $a \in \mathbb{C}^{n}, c \in \mathbb{R}^{n}, b \in C$ with $\Re b<0$, and $R$ is a function satisfying (33) (resp., (34));

(v) $g(x+y, t+s)-g(x, t) g(y, s)+f(x, t) f(y, s)=0$ for all $x, y \in \mathbb{R}^{n}, t, s>0$.

\section{Stability in Distributions and Hyperfunctions}

In this section we consider stability problems (8) and (9). Recall that the pullback $u \circ S$ in (8) and (9) is defined by

$$
\langle u \circ S, \varphi(x, y)\rangle=\left\langle u, \int \varphi(x-y, y) d y\right\rangle, \quad \varphi \in \mathscr{G}\left(\mathbb{R}^{2 n}\right) .
$$

In view of Definition 4 , it is easy to see that if $\varphi_{j}(x, y) \epsilon$ $\mathscr{G}\left(\mathbb{R}^{2 n}\right)$ is a sequence such that $\varphi_{j}(x, y) \rightarrow 0$ as $j \rightarrow \infty$, then $\int \varphi_{j}(x-y, y) d y \rightarrow 0$ in $\mathscr{G}\left(\mathbb{R}^{n}\right)$ as $j \rightarrow \infty$. Thus, we have $u \circ S \in \mathscr{G}^{\prime}\left(\mathbb{R}^{2 n}\right)$.

Theorem 16. Let $u, v \in \mathscr{G}^{\prime}\left(\mathbb{R}^{n}\right)$. Then $(v, u)$ satisfies (8) (resp., (9)) if and only if $(v, u)$ satisfies one of the following:

(i) v, u: bounded distributions (resp., bounded hyperfunction);

(ii) $v=e^{c \cdot x}, c \in \mathbb{C}^{n}$, and $u$ : bounded distributions (resp., bounded hyperfunction); 
(iii) $v=\left(e^{c \cdot x}+\lambda w_{0}\right) /\left(1-\lambda^{2}\right), u=\left(\lambda e^{c \cdot x}+w_{0}\right) /\left(1-\lambda^{2}\right)$ for some $c \in \mathbb{C}^{n}, \lambda \in \mathbb{C}$, and $w_{0} \in \mathscr{D}_{L^{\infty}}^{\prime}\left(\mathbb{R}^{n}\right)$ (resp., $\left.\mathscr{A}_{L^{\infty}}^{\prime}\left(\mathbb{R}^{n}\right)\right)$;

(iv) $v=(a \cdot x+1) e^{i c \cdot x}+w_{0}, u= \pm a \cdot x e^{i c \cdot x}+w_{0}$ for some $a \in \mathbb{C}^{n}, c \in \mathbb{R}^{n}$, and $w_{0} \in \mathscr{D}_{L^{\infty}}^{\prime}\left(\mathbb{R}^{n}\right)\left(\right.$ resp., $\left.\mathscr{A}_{L^{\infty}}^{\prime}\left(\mathbb{R}^{n}\right)\right) ;$

(v) $v=(a \cdot x+1) e^{c \cdot x}, u= \pm a \cdot x e^{c \cdot x}$ for some $a, c \in \mathbb{C}^{n}$;

(vi) $v=e^{c \cdot x}[\cos (a \cdot x)+\lambda \sin (a \cdot x)], u= \pm e^{c \cdot x} \sqrt{\lambda^{2}+1} \sin (a$. $x)$ for some $a, c \in \mathbb{C}^{n}, \lambda \in \mathbb{C}$.

Proof. We first prove the inequality; there exist $C>0, N>0$, and $d>0$ (resp., for every $\epsilon>0$ there exists $C_{\epsilon}>0$ ) such that

$$
\begin{aligned}
& |\widetilde{v}(x+y, t+s)-\widetilde{v}(x, t) \widetilde{v}(y, s)+\widetilde{u}(x, t) \tilde{u}(y, s)| \\
& \quad \leq C\left(\frac{1}{t}+\frac{1}{s}\right)^{N}+d \quad\left(\text { resp., } C_{\epsilon} e^{\epsilon(1 / t+1 / s)}\right),
\end{aligned}
$$

where $\widetilde{u}, \widetilde{v}$ are the Gauss transforms of $u, v$, respectively, given in Lemmas 6 and 7. Convolving the tensor product $E_{t}(x) E_{s}(y)$ of $n$-dimensional heat kernels in the left hand side of (8), in view of the semigroup property $\left(E_{t} * E_{s}\right)(x)=$ $E_{t+s}(x)$ of the heat kernel we have

$$
\begin{aligned}
& {\left[(v \circ S) *\left(E_{t}(\xi) E_{s}(\eta)\right)\right](x, y)} \\
& \quad=\left\langle v_{\xi}, \int E_{t}(x-\xi+\eta) E_{s}(y-\eta) d \eta\right\rangle \\
& \quad=\left\langle v_{\xi},\left(E_{t} * E_{s}\right)(x+y-\xi)\right\rangle \\
& \quad=\widetilde{v}(x+y, t+s) .
\end{aligned}
$$

Similarly we have

$$
\begin{aligned}
{\left[(v \otimes v) *\left(E_{t}(\xi) E_{s}(\eta)\right)\right](x, y) } & =\widetilde{v}(x, t) \widetilde{v}(y, s), \\
{\left[(u \otimes u) *\left(E_{t}(\xi) E_{s}(\eta)\right)\right](x, y) } & =\widetilde{u}(x, t) \widetilde{u}(y, s),
\end{aligned}
$$

where $\widetilde{u}(x, t), \widetilde{v}(x, t)$ are the Gauss transforms of $u, v$, respectively.

Let $w:=v \circ S-v \otimes v-u \otimes u$. Then $w \in \mathscr{D}_{L^{\infty}}^{\prime}\left(\mathbb{R}^{2 n}\right)$ (resp., $\mathscr{A}_{L^{\infty}}^{\prime}\left(\mathbb{R}^{2 n}\right)$ ). First, we suppose that $w \in \mathscr{D}_{L^{\infty}}^{\prime}\left(\mathbb{R}^{2 n}\right)$. Using Lemma 8 and Proposition 10 we have

$$
\begin{aligned}
& \left|\left[w *\left(E_{t}(\xi) E_{s}(\eta)\right)\right](x, y)\right| \\
& \quad \leq \sum_{|\alpha| \leq p}\left|\left[\partial^{\alpha} f_{\alpha} *\left(E_{t}(\xi) E_{s}(\eta)\right)\right](x, y)\right| \\
& \quad \leq \sum_{|\alpha| \leq p}\left|\left[f_{\alpha} * \partial_{\xi, \eta}^{\alpha}\left(E_{t}(\xi) E_{s}(\eta)\right)\right](x, y)\right| \\
& \quad \leq \sum_{|\alpha| \leq p}\left\|f_{\alpha}\right\|_{L^{\infty}}\left\|\partial_{\xi, \eta}^{\alpha}\left(E_{t}(\xi) E_{s}(\eta)\right)\right\|_{L^{1}} \\
& \quad \leq C_{1} \sum_{|\beta|+|\gamma| \leq p}\left\|\partial_{\xi}^{\beta} E_{t}(\xi)\right\|_{L^{1}}\left\|\partial_{\eta}^{\gamma} E_{s}(\eta)\right\|_{L^{1}} \\
& \quad \leq C_{2} \sum_{|\beta|+|\gamma| \leq p} t^{-(n+|\beta|) / 2} s^{-(n+|\gamma|) / 2} \\
& \quad \leq C\left(\frac{1}{t}+\frac{1}{s}\right)^{N}+d,
\end{aligned}
$$

where $N=n+p / 2$ and the constants $C$ and $d$ depend only on $p$. Secondly we suppose that $w \in \mathscr{A}_{L^{\infty}}^{\prime}\left(\mathbb{R}^{2 n}\right)$. Then, by Proposition 10 we have

$$
\begin{aligned}
& \left\|\Delta^{k}\left(E_{t}(\xi) E_{s}(\eta)\right)\right\|_{L^{1}} \\
& \leq \sum_{|\alpha|=k} \frac{k !}{\alpha !}\left\|\partial^{2 \alpha}\left(E_{t}(\xi) E_{s}(\eta)\right)\right\|_{L^{1}} \\
& \leq \sum_{|\beta|+|\gamma|=k} \frac{k !}{\beta ! \gamma !}\left\|\partial_{\xi}^{2 \beta} E_{t}(\xi)\right\|_{L^{1}}\left\|\partial_{\eta}^{2 \gamma} E_{s}(\eta)\right\|_{L^{1}} \\
& \leq \sum_{|\beta|+|\gamma|=k} \frac{k !(2 \beta) !^{1 / 2}(2 \gamma) !^{1 / 2} M^{2 k}}{\beta ! \gamma !} t^{-n / 2-|\beta|} s^{-n / 2-|\gamma|} \\
& \leq \sum_{|\beta|+|\gamma|=k} k !(2 M)^{2 k} t^{-n / 2-|\beta|} s^{-n / 2-|\gamma|} \\
& \leq k !(2 \sqrt{n} M)^{2 k}\left(\frac{1}{t}+\frac{1}{s}\right)^{n+k} .
\end{aligned}
$$

Now, by Lemma 9 we have

$$
\begin{aligned}
\mid[w & \left.*\left(E_{t}(\xi) E_{s}(\eta)\right)\right](x, y) \mid \\
\leq & \sum_{k=0}^{\infty}\left\|a_{k}\left(\Delta^{k} g\right) *\left(E_{t}(\xi) E_{s}(\eta)\right)\right\|_{L^{\infty}} \\
& +\left\|h *\left(E_{t}(\xi) E_{s}(\eta)\right)\right\|_{L^{\infty}} \\
\leq & \|g\|_{L^{\infty}} \sum_{k=0}^{\infty}\left\|a_{k} \Delta^{k}\left(E_{t}(\xi) E_{s}(\eta)\right)\right\|_{L^{1}} \\
& +\|h\|_{L^{\infty}}\left\|E_{t}(\xi) E_{s}(\eta)\right\|_{L^{1}} \\
\leq & C_{1} \sum_{k=0}^{\infty} \frac{1}{k !}\left(4 n M^{2} L\right)^{k}\left(\frac{1}{t}+\frac{1}{s}\right)^{n+k}+\|h\|_{L^{\infty}} \\
\leq & C_{2} \sum_{k=0}^{\infty} \frac{1}{k !} \epsilon^{k}\left(\frac{1}{t}+\frac{1}{s}\right)^{n+k}+\|h\|_{L^{\infty}} \\
\leq & C_{\epsilon} e^{\epsilon(1 / t+1 / s)},
\end{aligned}
$$

where $L$ is taken so that $4 n M^{2} L<\epsilon$ and the constant $C_{\epsilon}$ depends only on $w$ and $\epsilon$. Thus, we have inequality (57). Using Corollary 15 we obtain one of the following:

(I) both $\widetilde{v}$ and $\tilde{u}$ satisfy (33) (resp., (34));

(II) $\widetilde{v}(x, t)=e^{c \cdot x+b t}$ and $\widetilde{u}$ satisfies (33) (resp., (34)), where $c \in \mathbb{C}^{n}, b \in \mathbb{C}$

(III) $\widetilde{v}(x, t)=\left(e^{c \cdot x+b t}+\lambda R(x, t)\right) /\left(1-\lambda^{2}\right), \widetilde{u}(x, t)=$ $\left(\lambda e^{c \cdot x+b t}+R(x, t)\right) /\left(1-\lambda^{2}\right)$, where $c \in \mathbb{C}^{n}, b, \lambda \in \mathbb{C}$, and $R$ is a function satisfying (33) (resp., (34));

(IV) $\widetilde{v}(x, t)=(a \cdot x+1) e^{i c \cdot x+b t}+2 \widetilde{v}(0, t) e^{i c \cdot x+(1 / 2) b t}+R(x, t)$, $\pm \widetilde{u}(x, t)=a \cdot x e^{i c \cdot x+b t}+2 \widetilde{v}(0, t) e^{i c \cdot x+(1 / 2) b t}+R(x, t)$, where $a \in \mathbb{C}^{n}, c \in \mathbb{R}^{n}, b \in C$ with $\Re b<0$, and $R$ is a function satisfying (33) (resp., (34));

(V) $\widetilde{v}(x+y, t+s)-\widetilde{v}(x, t) \widetilde{v}(y, s)+\widetilde{u}(x, t) \widetilde{u}(y, s)=0$. 
Letting $t \rightarrow 0^{+}$in (I), (II) we get (i) and (ii), respectively. From (III) we have

$$
\widetilde{u}(x, t)-\lambda \widetilde{v}(x, t)=R(x, t) .
$$

Thus $R(x, t)$ is a solution of the heat equation. Letting $t \rightarrow$ $0^{+}$in (III) and using Lemma 6 (resp., Lemma 7) we have $R(x, t) \rightarrow w_{0}$ for some $w_{0} \in \mathscr{D}_{L^{\infty}}^{\prime}\left(\mathbb{R}^{n}\right)$ (resp., $\mathscr{A}_{L^{\infty}}^{\prime}\left(\mathbb{R}^{n}\right)$ ), which gives case (iii). Now we prove (iv). From (IV), since

$$
\tilde{v}(x, t) \pm \tilde{u}(x, t)=e^{i c \cdot x+b t}
$$

$e^{i c \cdot x+b t}$ is a solution of the heat equation. Thus we have $b=$ $-\left(c_{1}^{2}+\cdots+c_{n}^{2}\right):=-|c|^{2}$, where $c=\left(c_{1}, \ldots, c_{n}\right)$. Let

$$
\begin{aligned}
R_{1}(x, t)= & -2 i a \cdot c t e^{i c \cdot x-|c|^{2} t} \\
& +2 \widetilde{v}(0, t) e^{i c \cdot x+(1 / 2) b t}+R(x, t) .
\end{aligned}
$$

Then, from (IV) we have

$$
\begin{gathered}
\widetilde{v}(x, t)=(a \cdot x+2 i a \cdot c t+1) e^{i c \cdot x-|c|^{2} t}+R_{1}(x, t), \\
\pm \widetilde{u}(x, t)=(a \cdot x+2 i a \cdot c t) e^{i c \cdot x-|c|^{2} t}+R_{1}(x, t) .
\end{gathered}
$$

Thus $R_{1}(x, t)$ is a solution of the heat equation. Putting $\pm \widetilde{u}(x, t)=\widetilde{v}(x, t)-e^{i c \cdot x-|c|^{2} t}$ in (57) and using the triangle inequality we have

$$
\begin{gathered}
\left|\widetilde{v}(x+y, t+s)-\widetilde{v}(x, t) e^{i c \cdot y-|c|^{2} s}-\widetilde{v}(y, s) e^{i c \cdot x-|c|^{2} t}\right| \\
\left.\leq C\left(\frac{1}{t}+\frac{1}{s}\right)^{N}+d+1 \quad \text { (resp., } C_{\epsilon}^{\prime} e^{\epsilon(1 / t+1 / s)}\right) .
\end{gathered}
$$

By the continuity of $\widetilde{v}$, there exists $M>0$ such that $\mid \widetilde{v}(0, t+$ $1) \mid \leq M$ for all $t \in[0,1]$. Putting $x=y=0, s=1$ in (67) and using the triangle inequality we have

$$
\begin{array}{r}
|\widetilde{v}(0, t)| \leq e^{|c|^{2}}\left(|\widetilde{v}(0, t+1)|+|\widetilde{v}(0,1)| e^{-|c|^{2} t}\right. \\
\left.+C\left(\frac{1}{t}+1\right)^{N}+d\right) \\
\leq e^{|c|^{2}}\left(2 M+C\left(\frac{1}{t}+1\right)^{N}+d\right) \\
\leq C^{\prime} t^{-N} \quad\left(\text { resp., } C_{\epsilon}^{\prime} e^{\epsilon / t}\right)
\end{array}
$$

for all $t \in(0,1)$. From (65) and (68) we can see that $R_{1}$ satisfies (20) (resp., (22)). Letting $t \rightarrow 0^{+}$in (66) and using Lemma 6 (resp., Lemma 7) we get (iv). Finally, we consider case (V). Letting $t, s \rightarrow 0^{+}$in $(\mathrm{V})$ we obtain

$$
v \circ S-v \otimes v+u \otimes u=0 .
$$

Now in view of [23, Theorem 3.1], all solutions $(v, u)$ of $(69)$ are of the same form as the smooth solutions $(g, f)$ of the classical functional equation

$$
g(x+y)-g(x) g(y)+f(x) f(y)=0
$$

and are given by (iii), (v), or (vi) (see [37, page 180], [23, Theorem 4.2]). This completes the proof.
Now we consider Ulam problem (8) in the space of Schwartz tempered distributions. Recall that the following topological inclusions hold:

$$
\begin{gathered}
\mathscr{G} \hookrightarrow \mathcal{S} \hookrightarrow \mathscr{D}_{L^{1}}, \quad D_{L^{\infty}}^{\prime} \hookrightarrow \mathcal{S}^{\prime} \hookrightarrow \mathscr{G}^{\prime}, \\
\mathscr{G} \hookrightarrow \mathscr{A}_{L^{1}} \hookrightarrow \mathscr{D}_{L^{1}}, \quad \mathscr{D}_{L^{\infty}}^{\prime} \hookrightarrow \mathscr{A}_{L^{\infty}}^{\prime} \hookrightarrow \mathscr{G}^{\prime} .
\end{gathered}
$$

In view of the above topological inclusions, if $u, v \in$ $\mathcal{S}^{\prime}\left(\mathbb{R}^{n}\right)$ it is natural to consider the Ulam problem

$$
v \circ S-v \otimes v+u \otimes u \in \mathscr{D}_{L^{\infty}}^{\prime}\left(\mathbb{R}^{2 n}\right) .
$$

Theorem 17. Let $u, v \in \mathcal{S}^{\prime}\left(\mathbb{R}^{n}\right)$. Then $(v, u)$ satisfies (72) if and only if $(v, u)$ satisfies one of the following:

(i) $v, u$ : bounded distributions;

(ii) $v=(a \cdot x+1) e^{i c \cdot x}+w_{0}, u= \pm a \cdot x e^{i c \cdot x}+w_{0}$ for some $a \in \mathbb{C}^{n}, c \in \mathbb{R}^{n}$, and $w_{0} \in \mathscr{D}_{L^{\infty}}^{\prime}\left(\mathbb{R}^{n}\right)$.

Proof. Since $u, v \in \mathcal{S}^{\prime}\left(\mathbb{R}^{n}\right)$, we have $c=i a$ for some $a \in \mathbb{R}^{n}$ and cases (ii), (iii), and (vi) in Theorem 16 are reduced to case (i) and case (v) is contained in case (iv). This completes the proof.

Finally we discuss the stability of the following stability problem (see [24]):

$$
v \circ S-v \otimes v+u \otimes u \in L^{\infty}\left(\mathbb{R}^{2 n}\right),
$$

where $L^{\infty}\left(\mathbb{R}^{2 n}\right)$ denotes the space of bounded measurable functions on $\mathbb{R}^{2 n}$. For the proof we use the following lemma instead of Lemmas 6 and 7.

Lemma 18 (see [38, page 122]). Let $f(x, t)$ be a solution of the heat equation. Then $f(x, t)$ satisfies

$$
|f(x, t)| \leq M, \quad x \in \mathbb{R}^{n}, t \in(0,1)
$$

for some $M>0$, if and only if

$$
f(x, t)=\left(f_{0} * E_{t}\right)(x)=\int f_{0}(y) E_{t}(x-y) d y
$$

for some bounded measurable function $f_{0}$ defined in $\mathbb{R}^{n}$. In particular, $f(x, t) \rightarrow f_{0}(x)$ in almost everywhere $x \in \mathbb{R}^{n}$ as $t \rightarrow 0^{+}$.

Following the same approach in the proof of Theorem 16 we have

$$
|\widetilde{v}(x+y, t+s)-\widetilde{v}(x, t) \widetilde{v}(y, s)+\widetilde{u}(x, t) \widetilde{u}(y, s)| \leq C,
$$

where $C>0$ and $\tilde{u}, \widetilde{v}$ are the Gauss transforms of $u, v$. Now using Corollary 15 for $N=0$ and Lemma 18 we have the following.

Theorem 19. Let $u, v \in \mathscr{G}^{\prime}\left(\mathbb{R}^{n}\right)$. Then $(v, u)$ satisfies (76) if and only if $(v, u)$ satisfies one of the following: 
(i) v, u: bounded measurable functions;

(ii) $v=e^{c \cdot x}$ for some $c \in \mathbb{C}^{n}$, u: bounded measurable functions;

(iii) $v=\left(e^{c \cdot x}+\lambda B(x)\right) /\left(1-\lambda^{2}\right), u=\left(\lambda e^{c \cdot x}+B(x)\right) /\left(1-\lambda^{2}\right)$ for some $c \in \mathbb{C}^{n}, \lambda \in \mathbb{C}$, and $B \in L^{\infty}\left(\mathbb{R}^{n}\right)$;

(iv) $v=(a \cdot x+1) e^{i c \cdot x}+B(x), u= \pm a \cdot x e^{i c \cdot x}+B(x)$ for some $a \in \mathbb{C}^{n}, c \in \mathbb{R}^{n}$, and $B \in L^{\infty}\left(\mathbb{R}^{n}\right)$;

(v) $v=(a \cdot x+1) e^{c \cdot x}, u= \pm a \cdot x e^{c \cdot x}$, where $a, c \in \mathbb{C}^{n}$;

(vi) $v=e^{c \cdot x}[\cos (a \cdot x)+\lambda \sin (a \cdot x)], u= \pm e^{c \cdot x} \sqrt{\lambda^{2}+1} \sin (a$. $x)$ for some $a, c \in \mathbb{C}^{n}, \lambda \in \mathbb{C}$.

Let $f$ be a Lebesgue measurable function on $\mathbb{R}^{n}$ satisfying the inequality; for every $\epsilon>0$ there exists $C_{\epsilon}>0$ such that

$$
|f(x)| \leq C_{\epsilon} e^{\epsilon|x|^{2}}
$$

for all $x \in \mathbb{R}^{n}$. The function satisfying (73) is said to be an infraexponential function of order 2 . It is easy to see that every infraexponential function $f$ of order 2 defines an element of $\mathscr{G}^{\prime}\left(\mathbb{R}^{n}\right)$ via the correspondence

$$
\langle f, \varphi\rangle=\int f(x) \varphi(x) d x
$$

for $\varphi \in \mathscr{G}$. Thus as a direct consequence of Theorem 19 we have the following.

Theorem 20. Let $g, f$ be infraexponential functions of order 2. Then $(g, f)$ satisfies the inequality; there exists $C>0$ such that

$$
\|g(x+y)-g(x) g(y)+f(x) f(y)\|_{L^{\infty}\left(\mathbb{R}^{2 n}\right)} \leq C
$$

if and only if $(g, f)$ satisfies one of the following in almost everywhere sense:

(i) $g, f$ : bounded measurable functions;

(ii) $g(x)=e^{c \cdot x}$ for some $c \in \mathbb{C}^{n}, f$ : bounded measurable functions;

(iii) $g(x)=\left(e^{c \cdot x}+\lambda B(x)\right) /\left(1-\lambda^{2}\right), f(x)=\left(\lambda e^{c \cdot x}+\right.$ $B(x)) /\left(1-\lambda^{2}\right)$ for some $c \in \mathbb{C}^{n}, \lambda \in \mathbb{C}$, and $B \in$ $L^{\infty}\left(\mathbb{R}^{n}\right)$;

(iv) $g(x)=(a \cdot x+1) e^{i c \cdot x}+B(x), f(x)= \pm a \cdot x e^{i c \cdot x}+B(x)$ for some $a \in \mathbb{C}^{n}, c \in \mathbb{R}^{n}$, and $B \in £^{\infty}\left(\mathbb{R}^{n}\right)$;

(v) $g(x)=(a \cdot x+1) e^{c \cdot x}, f(x)= \pm a \cdot x e^{c \cdot x}$ for some $a, c \in$ $\mathbb{C}^{n}$;

(vi) $g(x)=e^{c \cdot x}[\cos (a \cdot x)+\lambda \sin (a \cdot x)], f(x)=$ $\pm e^{c \cdot x} \sqrt{\lambda^{2}+1} \sin (a \cdot x)$ for some $a, c \in \mathbb{C}^{n}, \lambda \in \mathbb{C}$.

\section{Conflict of Interests}

The authors declare that there is no conflict of interests regarding the publication of this paper.

\section{Acknowledgments}

This work was supported by the Basic Science Research Program through the National Foundation of Korea (NRF) funded by the Ministry of Education Science and Technology (MEST) (no. 2012R1A1A008507). The authors express their deep thanks to the referees for the valuable comments on the paper.

\section{References}

[1] S. M. Ulam, Problems in Modern Mathematics, chapter 6, John Wiley \& Sons, New York, NY, USA, 1964.

[2] D. H. Hyers, "On the stability of the linear functional equation," Proceedings of the National Academy of Sciences of the United States of America, vol. 27, no. 4, pp. 222-224, 1941.

[3] D. H. Hyers, G. Isac, and Th. M. Rassias, Stability of Functional Equations in Several Variables, Birkhäuser, 1998.

[4] T. Aoki, "On the stability of the linear transformation in Banach spaces," Journal of the Mathematical Society of Japan, vol. 2, pp. 64-66, 1950.

[5] D. G. Bourgin, "Classes of transformations and bordering transformations," Bulletin of the American Mathematical Society, vol. 57, pp. 223-237, 1951.

[6] D. G. Bourgin, "Multiplicative transformations," Proceedings of the National Academy of Sciences of the United States of America, vol. 36, pp. 564-570, 1950.

[7] S. Czerwik, Stability of Functional Equations of Ulam-HyersRassias Type, Hadronic Press, Palm Harbor, Fla, USA, 2003.

[8] I. S. Fenyö, “On an inequality of P.W. Cholewa," in General Inequalities 5, vol. 80 of International Series of Numerical Mathematics, pp. 277-280, Birkhäuser, Basel, Switzerland, 1987.

[9] G. L. Forti, "Hyers-Ulam stability of functional equations in several variables," Aequationes Mathematicae, vol. 50, no. 1-2, pp. 143-190, 1995.

[10] S.-M. Jung, Hyers-Ulam-Rassias Stability of Functional Equations in Nonlinear Analysis, Springer, New York, NY, USA, 2011.

[11] J. M. Rassias, "On approximation of approximately linear mappings by linear mappings," Journal of Functional Analysis, vol. 46, no. 1, pp. 126-130, 1982.

[12] J. M. Rassias, "On approximation of approximately linear mappings by linear mappings," Bulletin des Sciences Mathématiques, vol. 108, pp. 445-446, 1984.

[13] J. M. Rassias, "Solution of a problem of Ulam," Journal of Approximation Theory, vol. 57, no. 3, pp. 268-273, 1989.

[14] Th. M. Rassias, "On the stability of functional equations in Banach spaces," Journal of Mathematical Analysis and Applications, vol. 251, no. 1, pp. 264-284, 2000.

[15] T. M. Rassias, "On the stability of the linear mapping in Banach spaces," Proceedings of the American Mathematical Society, vol. 72, no. 2, pp. 297-300, 1978.

[16] K. Ravi and M. Arunkumar, "On the Ulam-Gavruta-Rassias stability of the orthogonally Euler-Lagrange type functional equation," International Journal of Applied Mathematics \& Statistics, vol. 7, pp. 143-156, 2007.

[17] L. Székelyhidi, "The stability of the sine and cosine functional equations," Proceedings of the American Mathematical Society, vol. 110, no. 1, pp. 109-115, 1990.

[18] L. Székelyhidi, “The stability of d'Alembert type functional equations," Acta Scientiarum Mathematicarum, vol. 4, pp. 313$320,1982$. 
[19] L. Schwartz, Théorie des Distributions, Hermann, Paris, France, 1966.

[20] L. Hörmander, The Analysis of Linear Partial Differential Operators I, Springer, New York, NY, USA, 1983.

[21] I. M. Gelfand and G. E. Shilov, Generalized Functions IV, Academic Press, New York, NY, USA, 1968.

[22] J. Chung, "Stability of exponential equations in Schwarz distributions," Nonlinear Analysis: Theory, Methods \& Applications, vol. 69, no. 10, pp. 3503-3511, 2008.

[23] J.-Y. Chung, "A functional equation of Aczél and Chung in generalized functions," Advances in Difference Equations, vol. 2008, Article ID 147979, 11 pages, 2008.

[24] J. Chung and J. Chang, "On the stability of trigonometric functional equations in distributions and hyperfunctions," Abstract and Applied Analysis, vol. 2013, Article ID 275915, 12 pages, 2013.

[25] J. Chang and J. Chung, "Stability of trigonometric functional equations in generalized functions," Journal of Inequalities and Applications, vol. 2010, Article ID 801502, 12 pages, 2010.

[26] J. Chang and J. Chung, "The stability of the sine and cosine functional equations in Schwartz distributions," Bulletin of the Korean Mathematical Society, vol. 46, no. 1, pp. 87-97, 2009.

[27] J. A. Baker, "Distributional methods for functional equations," Aequationes Mathematicae, vol. 62, no. 1-2, pp. 136-142, 2001.

[28] J. A. Baker, "On a functional equation of Aczél and Chung," Aequationes Mathematicae, vol. 46, no. 1-2, pp. 99-111, 1993.

[29] J. A. Baker, "Functional equations, tempered distributions and Fourier transforms," Transactions of the American Mathematical Society, vol. 315, no. 1, pp. 57-68, 1989.

[30] J. Chung, S.-Y. Chung, and D. Kim, "A characterization for Fourier hyperfunctions," Publications of the Research Institute for Mathematical Sciences, vol. 30, no. 2, pp. 203-208, 1994.

[31] J. Chung, S.-Y. Chung, and D. Kim, "Une caractérisation de lespace de Schwartz," Comptes Rendus de l'Académie des Sciences-Series I-Mathematics, vol. 316, pp. 23-25, 1993.

[32] S.-Y. Chung, D. Kim, and E. G. Lee, "Periodic hyperfunctions and Fourier series," Proceedings of the American Mathematical Society, vol. 128, no. 8, pp. 2421-2430, 2000.

[33] T. Matsuzawa, "A calculus approach to hyperfunctions. II," Transactions of the American Mathematical Society, vol. 313, no. 2, pp. 619-654, 1989.

[34] I. M. Gelfand and G. E. Shilov, Generalized Functions. Vol. 2. Spaces of Fundamental and Generalized Functions, Academic Press, New York, NY, USA, 1968.

[35] S.-Y. Chung, "An heat equation approach to distributions with Lp growth," Communications of the Korean Mathematical Society, vol. 9, no. 4, pp. 897-903, 1994.

[36] J. Chung and D. Kim, "Ulam problem for the sine addition formula in hyperfunctions," Publications of the Research Institute for Mathematical Sciences, vol. 50, no. 2, pp. 227-250, 2014.

[37] J. Aczel, Lectures on Functional Equations and Their Applications, Mathematics in Science and Engineering, Vol. 19, Academic Press, New York, NY, USA, 1966.

[38] D. V. Widder, The Heat Equation, Academic Press, New York, NY, USA, 1975. 


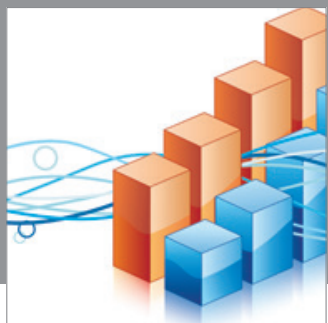

Advances in

Operations Research

mansans

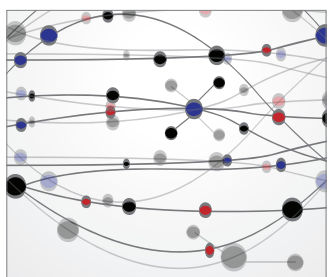

The Scientific World Journal
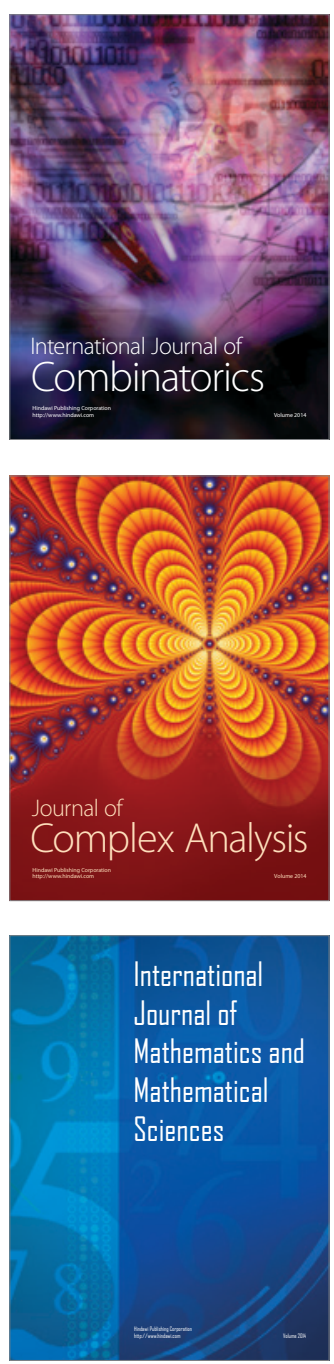
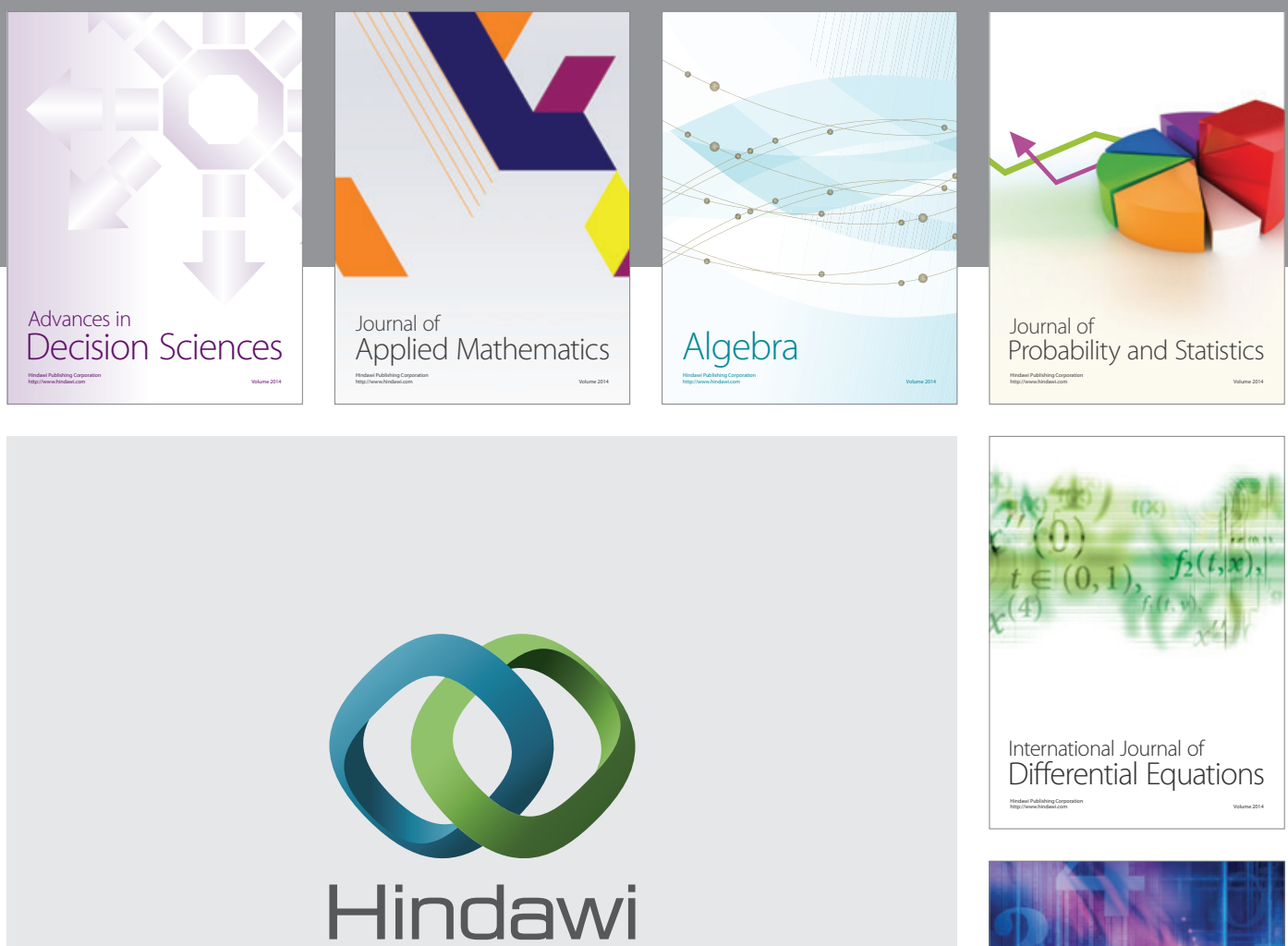

Submit your manuscripts at http://www.hindawi.com
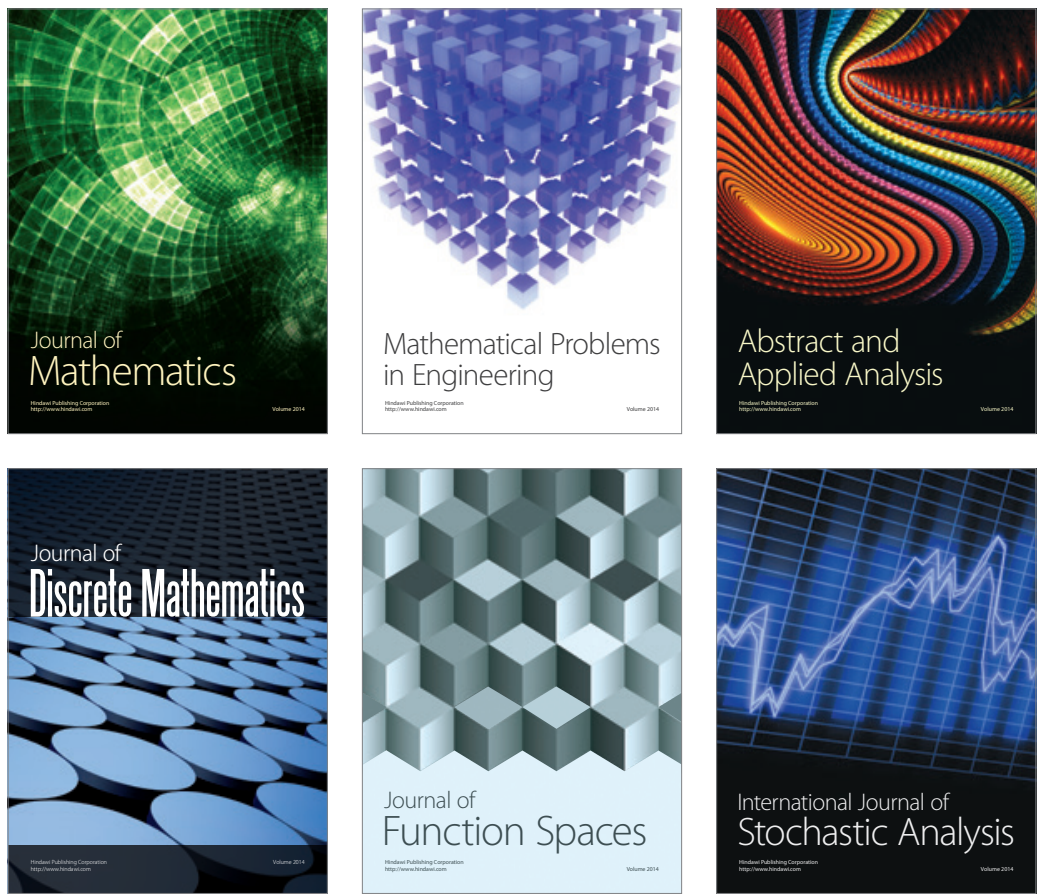

Journal of

Function Spaces

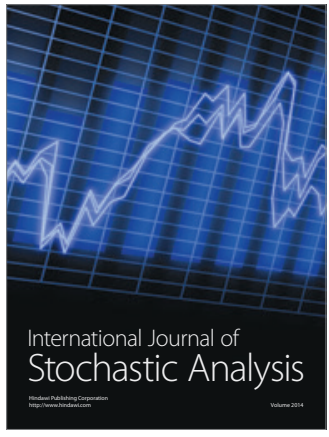

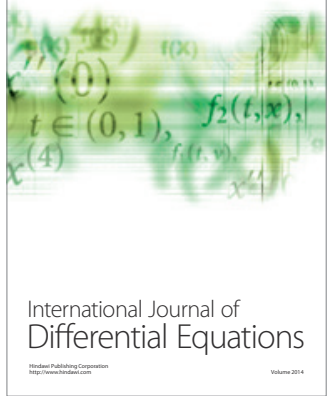
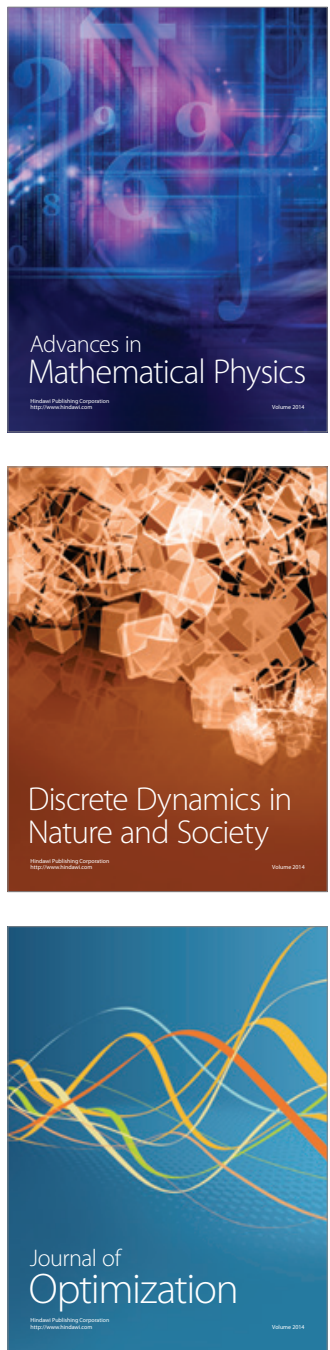